\title{
Sistematización de prácticas pedagógicas significativas en la carrera de licenciatura en educación infantil
}

\author{
Ana M. Jiménez-Quintero \\ Facultad de Educación, Licenciatura en Educación Infantil, Corporación Universitaria Minuto de Dios, \\ Carrera 74 81C-05, Bogotá, D. C., Colombia. (correo-e: anam.jimenezq@uniminuto.edu) \\ * Autor a quien debe ser enviada la correspondencia
}

Recibido Nov. 6, 2019; Aceptado Ene. 6, 2020; Versión final Mar. 12, 2020, Publicado Ago. 2020

\begin{abstract}
Resumen
La investigación que se describe orienta el estudio de la práctica pedagógica presente en la licenciatura en Educación Infantil de la Corporación Universitaria Minuto de Dios UNIMINUTO de Colombia, virtual y distancia tradicional. El proceso de investigación es cualitativo y está acogido en la sistematización de experiencias. La información recolectada se realizó a través de narrativas de estudiantes en formación en el año 2018 y parte de un periodo académico del año 2019. Se dilucidaron diferentes comprensiones, teniendo en cuenta los contextos educativos en los que participa el educador infantil, así como también reflexiones en torno a sus experiencias educativas. Se concluye que las prácticas pedagógicas requieren de mayores construcciones a partir de su pertinencia en la formación de maestros a través de lecturas críticas de realidades en la infancia. Esto incluye diversas experiencias mediadas por el contexto, la apropiación del saber y sentido pedagógico en la educación para la infancia.
\end{abstract}

Palabras clave: infancia; práctica pedagógica; educación; educación a distancia

\section{Systematization of significant pedagogical practices in the bachelor degree in early childhood education}

\begin{abstract}
The aim of this investigation is to provide guidelines on the study of the pedagogical practice in the degree of Children's Pedagogy virtual UNIMINUTO and traditional distance at the University Corporation Minute of God in Colombia. This is a qualitative research process that uses the systematization of experiences. The information collected was obtained from narratives of students enrolled in the 2018 and 2019 (part) academic periods. The results show differences in understanding depending on the educational contexts in which the child educator participates and on the educator's reflections on their educational experiences. In conclusion, pedagogical practices require major constructions, given their relevance in teacher training, through critical readings of childhood realities. This includes experiences mediated by the context, the appropriation of knowledge, and the pedagogical sense in childhood education.
\end{abstract}

Keywords: childhood; pedagogical practice; education; distance education 


\section{INTRODUCCIÓN}

La infancia se ha considerado un importante campo de estudios, sobre todo en los procesos de formación de educadores infantiles, albergando construcciones que han permitido re-pensar la práctica pedagógica que orientan y fortalecen el trabajo en la educación inicial. (Ackesjö y Persson 2016) indican que parte del trabajo en la infancia ha sido el de promover la participación en los niños y niñas desde una pedagogía social, siendo un aspecto ineludible que debe hacer parte de la formación de maestros, en el que se asuma un vínculo inacabado a través del rol del contexto, a partir de una visión pedagógica que vincule el maestro y el estudiante. Asimismo, (Ackesjö 2014) indica que ha sido una generalidad en los maestros en educación inicial buscar definir su rol, la importancia del trabajo en la infancia y la relación con la escuela. (Kaplan y Herzog 2016) confieren la importancia de posibilitar procesos de aprendizaje por parte de los maestros en donde se relacione la calidad educativa, sobre todo acogiendo las diversas formas de aprender que se tiene en la educación inicial. En América Latina, promover la educación en la infancia, sugiere el reconocimiento del niño como sujeto social en el marco de los derechos humanos, así lo refiere (Arrabal y Lázaro 2013) quienes ilustran que dicha labor debe ser prioridad gracias a la voluntad política de los gobiernos. Un ejemplo claro de esta perspectiva, ha sido el abordaje acogido a nivel mundial de una educación para todos en clave de atención a la diversidad, contando con un reconocimiento y equidad de condiciones de acceso permanencia en el sistema educativo.

Asimismo (Henao y Gómez 2013) ubican las relaciones concurrentes entre política, niñez y vulnerabilidad a través de vínculos relacionales del contexto, en la que el desarrollo de niños y niñas no puede comprenderse ni agenciarse por fuera del contexto cultural, político y socio-económico, en el cual viven sus procesos de constitución de subjetividad e identidad mediado por condiciones estructurales de diversidad, desigualdad, violencias, emergencias y renegociaciones permanentes entre los actores y donde el maestro asume un lugar preponderante con su acción transformadora, lo que apunta a poner en el escenario de la educación un afinado vínculo con el entorno, a partir del reconocimiento de las realidades y sentidos que se viven cuando se piensa infancia. En correspondencia con lo anterior, parte de los desarrollos a tener en cuenta con la práctica pedagógica asumiendo el contexto, ha sido el de las aportaciones del ámbito político. Para el año 2017 el Ministerio de Educación Nacional colombiano ha conferido políticas que buscan tener en cuenta la apropiación de saberes desde la experiencia, que suscita pensar la educación y la diversidad educativa con la atención a la riqueza de grupos poblacionales del territorio nacional en concordancia al campo de estudios de las diferentes licenciaturas vigentes en el país.

Ser maestro, ha posibilitado un ejercicio pedagógico intencionado donde se hace imprescindible considerar la práctica pedagógica. (Noguera y Marín 2017) lo han estimado como un elemento central de la educación escolar y parte del ejercicio profesional de los maestros, donde en el fondo, intenta valorar las experiencias que se tejen al interior de los contextos pedagógicos y educativos, que construyen historias y que deben ser interpretadas desde las realidades del mundo social en la que se hace participe el educador. La Corporación Universitaria Minuto de Dios UNIMINUTO según lo informa el Proyecto Educativo Institucional del Sistema (2014) como institución educativa que forma profesionales en diversas áreas, incluyendo licenciados en Educación, promueve principios y criterios en la construcción de saberes pedagógicos, contando con los aportes de la pedagogía praxeológica, con apuestas metodológicas para investigar desde la práctica pedagógica. La propuesta orientadora cuenta con algunos principios que permiten dar lugar a la conciencia y el pensamiento crítico reflexivo alrededor de las construcciones que se tienen del mundo social a través de la praxis rodeada de significados y sentidos. En palabras discursivas de (Rodríguez 2019) es la praxeología parte de la acción humana que permite transformar la realidad y con ella, a las personas y sus contextos.

Otras movilizaciones académicas institucionales en UNIMINUTO entorno a la investigación de la práctica, han sido principalmente orientadas por (Vargas 2017) donde la pedagogía praxeológica tiene sus fundamentos en la naturaleza misma de la praxis "actividad sensata con miras a un resultado", en la competencia del actor para construir su propio actuar, convirtiéndose en autor, y en las limitaciones inherentes al contexto o las situaciones implicadas. Por consiguiente, el carácter reflexivo de la praxeología, procura poder observar y participar, no solo en la lectura de realidades en las que incide el maestro, sino también sus propias construcciones internas y su papel como investigador social en la que se cualifica diversos entramados de vivencias significativas genuinas a partir de la educación.

De ahí que, asumir comprensiones en la infancia debe hacer parte de los desarrollos en la interpretación de realidades que se tiene en este campo de estudio, en donde la práctica pedagógica se constituye como una práctica social y donde a propósito de este tema (Zuloaga 1999) vincula este elemento con el saber pedagógico, en la medida en que se puedan llegar a engendrar dominios mediante la aparición de nuevos objetos y conceptos, en controversias suscitadas desde un carácter histórico, también vinculadas con elementos propios de lo político. Además, Fierro (2000) brinda atributos a la importancia de la práctica pedagógica como parte de aquellas relaciones permanentes de carácter colectivo y cultural que se viven 
con otras personas, donde maestros y alumnos en función de la vida humana y la marcha de la sociedad, siendo condiciones que invitan a una indagación, exploración, descripción e interpretación frecuente de realidades en las que participa el maestro que educa para la infancia.

Por consiguiente, elementos como la práctica pedagógica en el maestro, cobra relevancia y sentido a nivel investigativo, donde a través de la práctica pedagógica en la educación infantil, se busca aportar a comprensiones que se tienen sobre la infancia en los discursos obtenidos a partir del contexto y las modalidades de práctica que vinculan la pedagogía praxeológica. Para ello, se fundamentan aspectos metodológicos con la Sistematización de Experiencias en la que según lo indica( Posada y Briceño 2016,) es entendida como un tipo de producción de conocimiento que se adelanta en las prácticas sociales. Este estudio se realiza a través de la investigación cualitativa realizada entre los años 2018 y 2019, contando con la participación de maestros en formación de la licenciatura en Educación Infantil a través de la recolección de información, emitida en líneas de tiempo, con un enfoque histórico hermenéutico y crítico social, de carácter narrativo, de análisis y aprendizajes de la experiencia en la práctica pedagógica. Los resultados obtenidos de la investigación arrojan la importancia de la recuperación de la memoria y reconstrucción histórica de las experiencias de la práctica pedagógica a partir sus modalidades de atención ejercida en el contexto, donde se identifica en el maestro en formación las percepciones acerca de las realidades que se tienen en la infancia, y en el que se hace indispensable la promoción de condiciones de carácter discursivo, mediante la apropiación del saber y sentido pedagógico en la educación infantil.

\section{LA PRÁCTICA PEDAGÓGICA}

La práctica pedagógica, es considerada un aspecto fundamental, en ella se hace posible conocer las realidades provistas desde la educación, vinculada con diversos aspectos influenciados por el contexto en donde se es capaz de ver, interactuar y dialogar en el mundo a través de las experiencias. (Arendt 1997) sugiere que estas formas de relación son provistas gracias a la acción donde nos insertamos en un mundo en el que ya están presentes otros. Lo anterior, sugiere entonces comprender que elementos como la "acción" vinculados a la práctica pedagógica deben ser provistos del discurso, del lenguaje, de la escucha y la interpelación que alude lo intersubjetivo, para llegar a la comprensión de ser, estar y de autoproclamarse de manera frecuente de un sujeto libre en el mundo.

Higgins (2010) indica que posturas de Arendt al ubicar la acción como centro para teorizar en la educación, puede tener diversas interpretaciones y dicotomías en lo que configura ser maestro, sobre todo a través de concepciones que se tienen de la praxis en un ejercicio verdadero de libertad, realizando una crítica sobre lo que confiere hoy día ser sujeto político en la educación. Para contextos latinoamericanos, constantes reflexiones han llevado la práctica pedagógica a una práctica social, contando con aproximaciones a corrientes como las pedagogías críticas, que permita interpretar las realidades y necesidades educativas de los contextos en los que participa el maestro. Freire citado (Ortega 2009) sugiere cuatro categorías que se encuentran implicadas en la práctica: los sujetos, el condicionamiento social del saber, el concepto de ideología y la intencionalidad humanizadora, siendo elementos constitutivos que aportan al sentido de ser maestro, en la relación del mundo social, preguntas que allí sugieren con los diversos fenómenos y condiciones que concurren en la práctica pedagógica de maestros.

Ubicando puntualmente la producción de conocimiento en la práctica pedagógica, la Corporación Universitaria Minuto de Dios UNIMINUTO en sus programas de formación profesional ha provisto reflexiones sobre las características del maestro en formación en educación infantil, que posea un carácter crítico y consciente de las necesidades que se viven en la infancia del contexto colombiano. Igualmente, convoca a pensar la práctica pedagógica contando con las orientaciones pedagógicas que permean la institucionalidad de UNIMINUTO, ubicada en la praxeología, definida como la posibilidad de adquisición de conocimiento sobre todo para los sujetos que participan en ella; que permita dilucidar acciones a través de lo que denomina "la praxis". En palabras de (Vargas 2011) se trata de enfatizar la necesidad de un enfoque praxeológico que dé sentido a las prácticas es, al mismo tiempo y precisamente, revalorizar la práctica que recupera su antiguo sentido de decisión.

En ese transitar de aporte a la producción de conocimiento en "la acción" vinculada a la práctica pedagógica del maestro, como institución en educación superior, se ha permitido avanzar en lazos cercanos a la episteme de la sistematización de experiencias a través de la "acción" y la "comprehensión de la práctica" en concordancia a los lineamientos normativos emitidos por el Ministerio de Educación Nacional por el cual se ajustan las característica específicas de calidad de los programas de licenciatura en la (Resolución 18583 de 2017 del Ministerio de Educación Nacional) donde se concibe la práctica pedagógica y educativa como la oportunidad de apropiar saberes que permitan integrar procesos de formación, conceptualización, observación, transposición, interacción o intervención, investigación, innovación y experimentación de escenarios escolares. En palabras de (Noguera y Marín 2017), la práctica debe reconocerse en el ejercicio pedagógico del maestro en una relación tensa entre elementos de(saberes, normas y sujetos) en una inacabada y constante deconstrucción y construcción de experiencias. 


\section{Redescubriendo la acción praxeológica}

Uno de los mayores desafíos que se tienen en la práctica pedagógica de los maestros en formación en educación infantil UNIMINUTO, ha sido la de conocer sus experiencias, a través de los contextos en los que participan en atención a la infancia, que permita promover sujetos competentes con compromiso social e investigativo que aporten a su perfil de licenciando UNIMINUTO. De allí que, contribuciones explícitas mediante la pedagogía praxeológica o visto también en un enfoque praxeológico (Vargas 2011) ubique la investigación en correspondencia con la práctica con la acción como parte del discurso, por el tipo de análisis que realiza y en el cual se pretende hacer que dicha praxis sea más consciente a nivel discursivo a través del lenguaje y el proceso social en el cual el actor o practicante esté implicado. La formación de maestros debe poseer apremiantes reflexiones sobre la práctica, finalmente en ella confluyen aprendizajes a través de las percepciones, emociones e interpretaciones de lo que ve, siente y lo cautiva. Parte de los estudios realizados por Bourdieu citado en la obra de (Wacquant y Akçaoğlu 2017) atribuyen como prelación el estudio del nodo conceptual de la práctica entendido a todo lo que hacen, piensan o sienten las personas en su contexto habitual o su medio. Pero más allá de eso, en un campo de estudios específico, se busca una interpretación crítica de la realidad mediante la relación de componentes teóricos -prácticos para el diseño y arquitectura de nuevos significados.

Recoger experiencias a través de la infancia como campo de estudio, es una construcción de indudables desafíos para el educador infantil. El discernimiento sobre el conocimiento que subyace desde su participación como sujeto social con postura crítica, ética, moral y de responsabilidad social, configurado con líneas de acción con fundamentos pedagógicos para la educación inicial permeado por políticas educativas, advierte situar la investigación en este campo a partir de un contexto social con suficientes retos que, de manera ineludible reconstruye y construye las subjetividades que aportan a la identidad del maestro a través de elementos constitutivos presentes en una pedagogía que adquiere un protagonismo participativo versátil y transformador, mediante la identificación, problematización, dinamización del conocimiento y la reflexión de los aprendizajes adquiridos de lo vivido. En palabras de (Lyndon et al., 2019) se trata de una investigación dirigida por la práctica, donde elementos de la praxeología sostienen la ética de manera centralizada; equilibra el poder proporcionando un enfoque participativo a la investigación, al tiempo que reconoce la multiplicidad de realidades percibidas y experiencias participativas. Asimismo, otros estudios, sugieren investigar a través de las prácticas en maestros para la atención en la primera infancia, (Winterbottom y Mazzocco 2016) confieren relevancia al acoger un aprendizaje praxeológico y académico, donde se reconozcan experiencias para una mejor y más efectiva pedagogía.

El concepto de infancia en su devenir histórico, ha traído consigo tensiones y transformaciones frente a lo que ha significado para la humanidad, el interés por el abordaje en este campo de estudio para la formación de licenciados en educación infantil y que ha constituido construcciones a través de la apropiación de conocimientos y saberes pedagógicos en torno al desarrollo biológico, social, económico, político y cultural en niños y niñas. En esta misma línea, parte de las preguntas e inquietudes que surgen en la infancia o infancias en un sentido más amplio y pluralista como lo ilustra el documento maestro de la Licenciatura en Educación Infantil (2017) que orienta los lineamientos del programa para la renovación y registro calificado ante el Ministerio de Educación Nacional, invita a entender la postura en diversas miradas, tomando de referente el devenir histórico contemporáneo permeado por una interpretación genuina, propia de la historicidad de maestro como sujeto social, pero también rodeada con tensiones de diálogo institucionales y de políticas educativas que influyen en los contextos comunitarios del territorio nacional y su labor de educar, acogiendo de manera significativa elementos rodeados de sentidos, significados y apropiaciones gracias a la práctica hacia la transformación de realidades desde la acción pedagógica.

Al respecto (Sousa 2019) indica que, para lograr un adecuado proceso de atención en la infancia, se debe tener un carácter participativo donde se reconozcan las voces de los diferentes actores que hacen parte del proceso educativo basado en el contexto, que busque crear una cultura pedagógica que respete la participación de los niños, que los desafíe a "ser" más, a pensar más, a hacer más, a aprender más y a narrar más. Esta consideración en particular, ubica pensar en la infancia en las subjetividades que se tejen y abrazan los procesos de aprendizaje, identidad y desarrollo social donde se constituyen los niños y las niñas, distantes de atributos homogeneizadores y normalizadores y donde por el contrario, procura en una perspectiva más crítica, la posibilidad de ser escuchado y valorado con sus puntos de vista, siendo capaz de ser un sujeto autónomo y participativo de su propio aprendizaje, contando con el apoyo garante del maestro por medio del registro y voz de las experiencias que recojan diversidad de narraciones que promuevan aprendizajes para comprender la infancia.

Por consiguiente, las construcciones actuales en la infancia, acuden a estudiarla en variados entramados donde prima la configuración política de un sujeto de derechos, sobre todo en países latinoamericanos permeados por la desigualdad social. En materia de educación países como Colombia, mantienen las inequidades, las cuales siguen siendo profundas y reproducen las condiciones de exclusión en el territorio nacional, considerando poblaciones víctimas del conflicto armado, grupos culturalmente diversos entre las 
que se encuentran poblaciones con discapacidad, migrantes, indígenas, afrodescendientes, entre otros. (Ainscow 2012) confiere la importancia que tiene el maestro y la escuela en la función de promover sistemas educativos inclusivos y la posibilidad de imaginar lo que podría conseguirse a partir del sentido de responsabilidad compartida y colaborativa para hacerlo realidad.

De este modo, parte de los desarrollos que tiene la formación en educación infantil es ser consciente de las realidades vividas en el país, que a manera de reflexión sugieren dos elementos a considerar. En primer lugar, a través de las representaciones sociales invisibles, visibles, atraídas y transformadas desde los diversos contextos sociales en el que la infancia ha hecho parte y como segundo momento, reconocer el protagonismo de la educación en dichas construcciones, a partir de acercamiento y /o distancias de las construcciones frecuentes de análisis alrededor del niño y la niña somo sujeto social.

Estudios documentales de (Gómez y Alzate 2014) sobre la infancia contemporánea concluyen que al final de dos siglos de evolución, el niño o niña de hoy en día es actor social. En este sentido, parte de los compromisos y retos que la educación inicial sugieren es precisamente el reconocimiento de la infancia en esta ubicación, valorando la infancia en sus formas de producción de sujetos que han hecho parte de una historicidad social y que en lo político los ubica como sujetos de derechos y deberes de especial protección constitucional por parte del estado.

Parte de las provocaciones de los estudios en la infancia han sido las de descentralizar aspectos positivistas en las prácticas pedagógicas como una forma de investigar en la acción, que confiere las relaciones con los contextos educativos y donde subyacen preguntas frecuentes con el trabajo en la educación inicial alrededor de lo que se espera lograr de manera natural - biológica, contando con aprendizajes normalizadores en el paso provisional por el preescolar que se tiene en la infancia. (Sousa 2019) indica que parte de ese camino potenciador de reconocimiento y de valor donde participan los niños y las niñas, ha de permitir acudir a estrategias pedagógicas e investigativas praxeológicas basados en la documentación, que resulta ser fundamental para ver, reflexionar, comprender y transformar la pedagogía desarrollada con los niños. Por consiguiente, es fundamental que el maestro pueda asumir una práctica de sentidos, saberes, significados alrededor de la educación infantil. (Paredes 2014) citando la obra de Hugo Zemelman acude a un elemento dualista entre realidad y sujetos, así como entre sujetos y realidad con la importancia de la subjetividad, que permita ubicar a partir de una historicidad su dinámica social y formas de relación en perspectivas amplias que se tejen en ella. En este sentido, se concibe que parte de los principios orientadores de asumir y entender la infancia desde la educación, se encuentran permanentemente dispuestos en el vínculo intersubjetivo y dinamizador de los colectivos sociales que participen en ella.

\section{METODOLOGÍA}

Se trata de una modalidad de investigación cualitativa, que asume fundamentos de la investigación social y corrientes del pensamiento latinoamericano como la sistematización de experiencias, la cual busca reconstruir de manera analítica y reflexiva las experiencias con el fin de interpretarlas y comprenderlas, donde el investigador busca producir y aportar al conocimiento desde la identificación de diversos fenómenos sociales evidentes en los contextos educativos de la práctica pedagógica del educador infantil, teniendo presente los sentidos y apropiación del saber pedagógico que se tiene en la educación para la infancia.

En la educación popular (Mejía 2015) indica que, a través de la sistematización de experiencias se ratifica su valor educativo, pero también lo político, que contribuye al desarrollo de la educación transformadora y liberadora, (Torres 2014) propone que esta alternativa investigativa, busca contribuir a la producción de subjetividades, y la transformación de realidades en una perspectiva de saberes que se centran en las relaciones vinculantes que se tengan con diversos colectivos sociales.

La sistematización de experiencias, asume elementos metodológicos de la fenomenología, toda vez que busca estudiar fenómenos subjetivos rodeados de sentido y donde la práctica pedagógica asume un lugar preponderante en la formación de maestros a través de las diversas percepciones que se tejen alrededor de su labor en la infancia a partir de los contextos educativos. En palabras de (Van Manen 2017) la fenomenología es el intento sistemático de descubrir y describir las estructuras de significado internas de la experiencia vivida que se intuyen o se captan a través de un estudio de los detalles o casos en que se encuentran en la experiencia vivida. De otro lado la orientación metodológica, ha de fortalecer la interpretación, la cual no debe ser mecánica, sino basada en ideas y perspectivas más allá de la descripción simple o literal (Miner 2012).

Lo anterior, ha de permitir reconocer la importancia de las relaciones dialógicas y reflexivas de los participantes, sobre todo desde lo significativo que podrían llegar a ser las prácticas pedagógicas de los maestros en formación, ubicados en el estudio de la infancia y acogiendo un ciclo de vida que requiere de permanentes problematizaciones con una memoria histórica, conciencia presente y un anhelo de futuro o 
futuros deseados que orientan algunas miradas fundamentales sobre el sentido de ser maestro y lo que puede proyectar somo sujeto en la educación.

La investigación se ubica en la Corporación Universitaria Minuto de Dios UNIMIMINUTO modalidad virtual y distancia, en su programa de Licenciatura en Educación Infantil en la ciudad de Bogotá en los periodos 2018 y 2019, donde participaron maestros en formación vinculados a la práctica orientada por la pedagogía praxeológica que fundamenta la acción. La (Figura 1) presenta la ruta de sistematización de las prácticas pedagógicas significativas, donde de manera inicial se ubica el contexto de las experiencias, para luego reconstruirlas con las fases de observación, formación y profundización correspondiente a las modalidades de prácticas pedagógicas de séptimo, octavo y noveno en el periodo académico respectivamente.
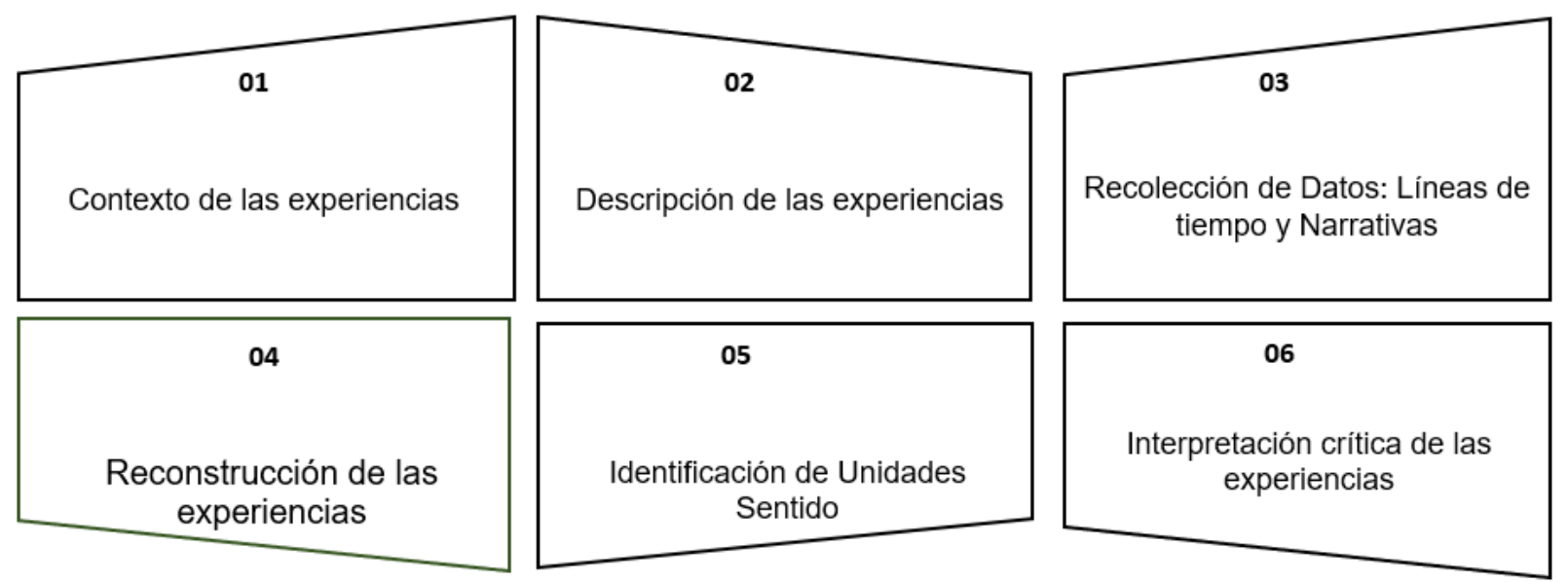

Fig. 1: Ruta metodológica e interpretativa de las prácticas pedagógicas significativas basado en el enfoque fenomenológico de Van Manen (2017)

Posterior a ello, se asumirán narrativas sobre líneas de tiempo como la posibilidad de capturar experiencias individuales y colectivas del proceso vivido. Para (Creswell 2013) las narrativas permiten recopilar sus historias, informar experiencias individuales y ordenar cronológicamente el significado de esas experiencias, donde se sitúan historias, situaciones personales, culturales, educativas y del contexto histórico. Por último, se realiza la identificación de las unidades de sentido presentes en las experiencias que dio lugar a la interpretación crítica a través de los hallazgos encontrados.

\section{RESULTADOS}

Este apartado visibiliza los hallazgos que suponen los momentos a considerar desde la Sistematización de Experiencias a través de la ubicación y descripción del contexto en el que se desarrolla la práctica pedagógica de los maestros en formación de la licenciatura en Educación Infantil, como forma de explicitar el fenómeno de estudio alrededor de la infancia y que de manera ineludible influye en su proceso formativo. Posterior a ello, se procurará a través de las narrativas y líneas de tiempo conocer diversas reflexiones alrededor de los diversos contextos donde se desarrollan las prácticas, sentido pedagógico y comprensión en infancias como parte de las unidades de sentido, que dieron lugar a la interpretación crítica de la experiencia.

\section{Caracterización del contexto de las experiencias significativas}

La caracterización de las experiencias significativas en las prácticas pedagógicas, cuenta con dos momentos. Inicialmente, en el contexto educativo que presenta la Corporación Universitaria Minuto de Dios UNIMINUTO para orientar sus prácticas hacia un trabajo praxeológico/investigativo y posterior a ello, las características de los contextos de las prácticas pedagógicas donde se encuentran vinculados los maestros en formación de la Licenciatura en Educación Infantil. De manera inicial, se puede decir que las prácticas pedagógicas se encuentran organizadas por fases que la componen: la práctica de observación en séptimo, la práctica de formación de octavo y la práctica de profundización de noveno periodo académico. Asimismo, la pedagogía praxeológica se encuentra inmersa a través de momentos otorgados mediante distintos fundamentos que hacen parte de la descripción de la experiencia. 
Tabla 1. Trabajo praxeológico/investigativo sobre la propia práctica profesional- adaptado Vargas (2011)

\begin{tabular}{|c|c|}
\hline Momentos & Fundamentos \\
\hline Ver & Análisis Crítico \\
\hline Juzgar & La interpretación \\
\hline Actuar & La re-elaboración operativa de la práctica \\
\hline Devolución creativa & Evaluación y prospectiva \\
\hline
\end{tabular}

La (tabla 1) indica que el participante que emerge en su práctica debe tener la posibilidad de recoger el mayor insumo posible de la observación de su práctica. Es un momento de planteamiento de preguntas, de iniciar un proceso experimental de aciertos y errores, pero creando conciencia sobre su relación con el mundo a través de la auto observación y la escucha participativa, en la observación praxeológica, la observación espontánea que se ubica en un contexto con los actores que participante. Luego, en el momento de juzgar, se hace necesario reconocer que se puede hacer con la recolección e insumo recogido en la fase del ver a través de la observación, en ese momento en la práctica se permite ahondar sobre autores y búsqueda documental que permita ubicar preguntas que orienten el sentido de la práctica con las experiencias, problematizaciones e interpretaciones de lo vivido y la visión que se tiene del mundo.

Posterior a ello, en el momento de actuar, se responde a la siguiente pregunta: ¿qué hacemos en concreto para mejorar la práctica? (Vargas 2011) donde a través del conocimiento del entorno y el contexto la acción es protagonista, y es donde se tienen claridades sobre los objetivos y propósitos que hacen parte de su praxis, al tener una lectura del en torno y sus necesidades, ineludiblemente se permite construcción de acciones, proyectos y evaluación de los mismos. Finalmente, se encuentra la devolución creativa, siendo la reflexión-acción la que origina reflexiones en torno a lo vivido, posee un componente prospectivo de lo vivido desde la praxis pedagógica, se trata de un proceso crítico que pretende proyectar transformar la experiencia pedagógica, con las ideas, la creación y la evaluación devolutiva de la práctica.

Cabe resaltar algunos aspectos de caracterización a partir de un documento de trabajo interno de Caracterización de la Práctica Pedagógica de la Licenciatura en Educación Infantil de la Corporación Universitaria Minuto de Dios del 2019 que hizo parte de esta investigación y en la que participaron 101 estudiantes, se pudo evidenciar que el $65 \%$ de los maestros en formación se encuentran en práctica con vínculo laboral o práctica laboral, mientras que el $32 \%$ fueron ubicadas en la modalidad de convenio que tiene UNIMINUTO con instituciones de la ciudad de Bogotá. Asimismo, la descripción de las experiencias informó que el $91 \%$ se encuentran realizando su práctica pedagógica en contextos urbanos, mientras que un $9 \%$ lo realizan en el área rural. Sobre el contexto relacionado a la atención en la infancia, un $67 \%$ de los maestros en formación indicaron que se encuentran vinculados en escenarios de educación inicial, mientras que un $28 \%$ realiza sus prácticas en contextos de educación formal donde la atención en preescolar se encuentra vinculada con la atención de estudiantes desde básica primaria y secundaria. Solo un $5 \%$ de los maestros en formación inciden en escenarios no formales en entidades no gubernamentales o sin ánimo de lucro, tales como fundaciones o entidades de trabajo comunitario. Otros de los aspectos estudiados del contexto fueron los relacionados al nivel socio-económico en las ciudades, donde según se indica el $41 \%$ de las instituciones en las que se realiza la práctica pedagógica pertenecen a la clase media, es decir personas que viven con un salario o dos salarios mínimos, un $21 \%$ a la clase baja con menos de un salario mínimo o en condiciones de pobreza y un $19 \%$ a la clase alta, con personas con un poder adquisitivo económico mayor.

Asimismo, dentro de la variedad de los contextos de la práctica pedagógica en la primera infancia que vigilan y regulan adecuadas prácticas de atención a niños y niñas, el $40 \%$ de los maestros en formación se encuentran en instituciones vigiladas por secretarias municipales y distritales, un $36 \%$ posee seguimiento desde la Secretaría de Integración Social en la ciudad de Bogotá, un 13\% se encuentran en otros, en relación a un 7\% que informan se encuentran con programas del Instituto Colombiano de Bienestar Familiar, siendo madres comunitarias o encontrándose laborando en Centros de Desarrollo Infantil. Lo anterior, ha permitido conocer las estancias territoriales de la ciudad de Bogotá y otros municipios cercanos que brindan seguimiento y veeduría a los procesos técnico, administrativo y pedagógico de las instituciones al servicio de la primera infancia.

\section{Unidades de sentido}

Las unidades de sentido se originaron gracias a la reconstrucción histórica de las experiencias mediante las líneas de tiempo y un ejercicio narrativo de los maestros en formación. Se utilizó una estructura organizativa por grupos que permitiera conocer el escenario de sus prácticas, experiencias vividas, aprendizajes y reflexiones. Alrededor de los aportes discursivos de las estudiantes, se establecieron aspectos relevantes de interpretación sobre el Sentido pedagógico y pensarse en la infancia- las infancias. En la primera unidad 
de sentido se hicieron visibles temas vinculados a las modalidades de atención educativa, las emociones en los maestros y el sentido de ser maestro. De otro lado, en la unidad de sentido de pensarse en la infancialas infancias se establecieron temas relacionados a percepciones que se tienen de la infancia, el contexto, la familia y la diversidad (Ver tabla 2)

Tabla 2: Unidades de sentido y temas relacionado a las percepciones que se tienen de la infancia

\begin{tabular}{|l|l|}
\hline Unidades de sentido & Temas \\
\hline \multirow{4}{*}{ Sentido pedagógico } & Modalidad de atención educativa \\
\cline { 2 - 2 } & Emociones en los maestros \\
\cline { 2 - 2 } & Sentido de ser maestro \\
\hline \multirow{4}{*}{ Pensarse en la infancia- infancias } & Percepciones en la infancia \\
\cline { 2 - 2 } & Contexto \\
\cline { 2 - 2 } & Familia \\
\cline { 2 - 2 } & Diversidad \\
\hline
\end{tabular}

\section{Sentido Pedagógico}

Esta unidad de sentido fue dispuesta como la apropiación de significados en el saber pedagógico de los maestros en formación en Educación Infantil y uno de los temas de mayor relevancia ha sido la modalidad de atención educativa, a partir de las narrativas de sus experiencias en las prácticas de observación, formación y profundización en los años 2018 y 2019. Las aportaciones coinciden alrededor de la ubicación y descripción de cada una de las experiencias con las instituciones de carácter público, privado o en los territorios, encontrándose relatos como "tener en cuenta si la institución es pública o privada, rural o urbana es clave a la hora del desarrollo y el proceso de cada niño, los modelos de atención cambian" (participante 1).

Asimismo, la comparación de los alcances interpretativos de los aprendizajes de la práctica pedagógica en relación a los estudiantes de práctica pedagógica laboral y la práctica pedagógica por convenio no remunerado, demuestran disímiles argumentaciones "Para mí ha sido muy enriquecedora la experiencia en la institución que asisto, bueno no ha sido solo desde los espacios de la práctica y el acompañamiento de la universidad, también ha sido una fortaleza que yo trabajo en este jardín infantil y debo conocer como son las orientaciones pedagógicas que brinda, la entidad que la vigila y las exigencias que solicitan para atender la primera infancia (participante 5). Otros relatos de práctica pedagógica laboral, han permitido ratificar modelos de atención vinculados con su cargo de maestro. "Yo soy auxiliar pedagógica, es decir yo solo asisto a las maestras de preescolar, pero fue para mí importante aprender a través de la práctica, observar, poder orientar una propuesta o idea pedagógica a través de una planeación y seguimiento, a pesar que no estaba desde mis funciones" (Participante 4)

Al contrario de las maestras en formación vinculadas a prácticas pedagógicas por convenio no remunerado, sus experiencias se muestran más cercanas a sus emociones, varias de las participantes no habían tenido experiencia en atención a la primera infancia y en sus narrativas reflejaron otras formas de sentido pedagógico. "Al principio confieso sentí miedo, no sabía que actuar y que decir, con el tiempo fue grato sentirme segura cuando empecé a relacionarme con las profes y los niños, aunque en mi planeación al principio me costó mucho, eso de aterrizar la teoría con la práctica" (Participante 10). Otras estudiantes presentan un sentido de pertenencia y responsabilidad social en las condiciones visibles de vulnerabilidad de los contextos en los que laboran, inclusive en sus narrativas dejaron de lado el carácter pedagógico alrededor de modelos pedagógicos o formas de enseñar, para centrarse en las realidades que viven a diario "En un jardín público y más en el sector en el que trabajo hay mucha vulnerabilidad, uno puede encontrar niños hijos de prostitutas, o de habitantes de la calle, o drogadictos, es muy normal encontrar situaciones de abuso sexual o que un día la mamá al dejar a su niño le diga a uno, me quiero matar" (Participante 2)

El sentido de ser maestro para el perfil formativo en Educación Infantil de UNIMINUTO sugiere ser sensible en relación a los desarrollos sociales donde participa. Los significados construidos por los maestros en formación señalan la reconstrucción de experiencias en las fases de observación, formación y profundización de la práctica pedagógica a través de las líneas de tiempo. Desde la práctica de observación, los maestros indicaron que fue importante apropiarse de conocimientos y la oportunidad de conocer de manera más consciente las realidades de las instituciones a partir de sus modelos de atención pedagógica "uno muchas veces deja pasar cosas para atender a los niños, uno asume que todo está bien, pero un día te preguntas ¿será que ese niño si desayunó, dormiría bien? ¿será que las actividades que estoy implementando le ayudarán a su aprendizaje (participante 9). 
De manera siguiente, en la fase formativa y de profundización, de las prácticas de octavo y noveno semestre, en los maestros se observan aprendizajes desde la resignificación o reelaboración de la práctica a partir de sus posturas críticas e interpretativas de las experiencias. "Cuando se llega a una institución se debe uno adaptar al currículo, pero poco a poco vas definiendo metas; por ejemplo, lo que dicen algunas es cierto, asumimos lineamientos en la educación inicial, las actividades rectoras que nos permitan sentirnos más a gusto" (participante 3). De otro lado, la narrativa de una de las maestras en formación es notorio en el taller. "De que sirve muchas veces tener la disposición para enseñar cosas nuevas, si el colegio donde se labora, no te lo permite por tener un enfoque tradicional, pues le toca a uno hacer lo que ellos dicen porque se queda uno sin empleo" (Participante 5)

\section{Pensarse en la infancia- infancias}

La unidad de sentido denominada pensarse en la infancia-infancias, ha proyectado la ubicación en un aspecto más amplio en las infancias, en consonancia con miradas más plurales de interpretar este campo de estudios. De este modo, en las narrativas de los maestros en formación se evidencian temas alrededor: los contextos de las prácticas pedagógicas, las familias y por último la diversidad como parte de las visiones actuales en la educación inclusiva. La mayoría de los contextos educativos donde se lleva a cabo la práctica pedagógica, obedece a contextos socioeconómicos de clase media y media alta y algunos contextos más vulnerables desde los jardines infantiles de carácter público.

Para el grupo de participantes entender las realidades que se viven en su su práctica ha sido fundamental para comprender las infancias. Narrativas como las de la (participante 1) "los sitios donde realizamos nuestras prácticas es clave, las edades de los niños, quienes son sus familias, si es un contexto por ejemplo rural o urbano en el que trabajamos, pues sabemos que el contexto cultural es totalmente clave a la hora del desarrollo y el proceso de cada niño". Igualmente, la (participante 5) indica que en el contexto nacen las experiencias significativas, dentro de su hogar, en sus espacios comunes y nosotros tenemos que aprender a respetar a valorar potencializar eso que ellos viven". Otras participantes informan que las instituciones privadas cuentan con adecuada infraestructura y talento humano, pero que la problemática a veces se encuentra relacionada con las características de los padres y los modelos de familia de los que hacen parte los niños y las niñas.

Precisamente, la familia, ha sido uno de los temas de mayor relevancia en las narrativas para pensarse en las infancias. Los maestros en formación indican que, este aspecto es el que les ha generado mayores retos. Destacan su influencia en el desarrollo de los niños que relacionan las diferentes problemáticas sociales que se viven en cada uno de los contextos educativos y la labor de corresponsabilidad social por parte de las familias en atención a las infancias; Las maestras en formación que establecieron relaciones frecuentes con mamá, papá o cuidadores, presentan narrativas sobre la labor de cuidado en las infancias, encontrándose los siguientes relatos: "en la institución donde laboro los padres son participativos, conocen del modelo pedagógico, nosotros acostumbramos a desarrollar talleres de crianza a familias, hace parte de nuestro propósito institucional" (participante 5). Otra maestra en formación como la (participante 3) que indica que en ocasiones es complejo tener comunicación con las familias "los padres son muy sobreprotectores, permisivos, ausentes, donde la figura de autoridad para los niños en muchas ocasiones no son sus padres, sino otras personas que se encuentran bajo su cuidado".

Existe un campo indiscutible de estudios para pensar en las infancias y es la vulnerabilidad en la que hacen parte fenómenos como el abandono y el maltrato infantil, propio de la falta del fortalecimiento de vínculos a desarrollar en las familias. Autores como (Taibo et al, 2018) muestran que las graves vulneraciones de los derechos en la infancia, se deben a diversas variables del funcionamiento familiar, en donde se debe considerar el fortalecimiento de los buenos tratos, la afectividad y la expresión positiva de emociones en la familia. Voces ilustran lo siguiente: "Uno observa y sabe cuándo un niño es maltratado, ellos mismos lo cuentan, además lo expresan en el colegio, contamos con una ruta de atención para esos casos y muchas veces les quitan a los niños a los padres de familia, pero al otro día, uno podría encontrar otra problemática similar o peor" (participante 1).

Finalmente, las narrativas confieren más experiencias significativas sobre la diversidad, relatando historias alrededor de la educación inclusiva. En el último semestre de la práctica pedagógica de profundización, se les permitió a los maestros en formación, ahondar sobre las prácticas inclusivas a través de estudios de caso. De lo anterior, se desarrollan manifestaciones relevantes sobre la importancia de reconocer las diferencias que tienen todos los niños y niñas, que también se centra en una perspectiva de derechos. "Las políticas educativas son claras cuando nos dicen, debemos incluir desde la diversidad, hay muchos chiquitos que llegan sin un diagnóstico y es en el jardín infantil donde nos damos cuenta que algo pasa" (Participante 1). 


\section{DISCUSIÓN}

La sistematización de experiencias para este proceso investigativo asumió elementos metodológicos de la fenomenología como parte del enfoque de la investigación a partir de las experiencias y significados, acogiendo la identificación y la interpretación del contexto institucional frente a las realidades presentes evidentes en la práctica pedagógica en la formación de maestros de la Licenciatura en Educación Infantil. Para ello se hizo necesaria la ubicación y descripción del contexto pedagógico, en la que se muestra una labor de caracterización, las orientaciones pedagógicas institucionales establecidas por la Corporación Universitaria Minuto de Dios UNIMINUTO y su carácter relacional con la praxeología (teoría-práctica) a través de los momentos sugeridos En la (tabla 1). Luego desde el análisis crítico y la interpretación se hizo evidente en cada una de las narrativas de los participantes con atribución a una reelaboración operativa de la práctica en algunos casos. En relación a lo esperado sobre la evaluación de su propia práctica frente a las necesidades del contexto, fueron pocas sus participaciones. solo una participante evidencia prospectiva en la relación de su práctica y las políticas en los avances significativos en la educación.

En las experiencias también se describen, inquietudes frecuentes de los educadores infantiles en formación a partir de su práctica pedagógica, conforme al mundo social y la interpretación que tienen sobre propuestas de transformación hacia las infancias en relación al entorno, la implementación de acciones pedagógicas y la inacabada evaluación de los aprendizajes vividos. Para autores como (Wacquant y Akçaoğlu 2017) la práctica es un nodo de estudio conceptual que permite revalorar todo lo que hacen, piensan o sienten las personas en su mundo normal. Pero más allá de eso, desde un campo de estudios específico, se busca una interpretación crítica de la realidad mediante la relación de componentes teóricos - prácticos para el diseño y arquitectura de nuevos significados. De otro lado, los estudios en infancias para los maestros en formación en el contexto colombiano, contempla seguir avanzando en perspicacias sobre el desarrollo social de las niñas y los niños evocando a través de la memoria, las concepciones del pasado para traerlas al presente, confrontarlas con lo que se asume en cada escenario en cada significado visible en las prácticas pedagógicas, posibilitando el lugar que ocupa la etapa de la primera infancia en la condición humana. Para (Coy y Rojas 2020) es necesario reconocer su presencia y participación en la historia, para comprender mejor la complejidad y variedad de dinámicas que los han involucrado en las sociedades de su tiempo el trabajo con la infancia.

Al llegar a las unidades de significado las narrativas, difieren en las concepciones de la práctica pedagógica y sus modalidades, Para los maestros en formación la práctica laboral, es clara la apropiación del sentido pedagógico en las orientaciones pedagógicas alrededor de la atención de niños y el conocimiento de las políticas educativas. En el país existen diversas condiciones de atención a la primera infancia y dependen en gran medida del territorio y entidades gubernamentales de carácter educativo.

Sobre las emociones de los maestros, se observa como en el proceso constitutivo de las subjetividades del maestro a partir de sus narrativas, se hacen evidentes tensiones alrededor de las relaciones interpersonales que se viven en la escuela o frustraciones acerca de eventos relacionados con la injusticia, muchas veces sin respuestas en la línea interinstitucional, existiendo también desasosiego en función de las problemáticas sociales que se viven desde las experiencias en función de los derechos para la población infantil. De otro lado, a través del saber en función de las propuestas pedagógicas y didácticas a implementar para los procesos de enseñanza en niños y niñas, conlleva de manera constante a una confrontación para los maestros a la hora de educar; teniendo presente preguntas como ¿que enseñar? $O$ ¿cómo enseñar? $Y$ tener la satisfacción y goce por haber logrado sus objetivos de trabajo a través de sus prácticas pedagógicas.

La categoría de pensarse en la infancia-infancias ha sido reconstruida y apropiada en parte gracias a los fundamentos teóricos del programa. Así, al reconocer la mirada estrecha y limitada que tiene "infancia" en singular, lleva a que los profesores del programa y los licenciados en formación transformen la práctica e incorporen teorías que se traducen en investigación, formación y proyección social, y se relacione con la categoría de infancias en la diversidad, la pluralidad, los contextos, la particularidad, la subjetividad, la incertidumbre, la interdependencia, como aspectos connaturales a las infancias, según lo informa el Documento con fines de Acreditación del programa de la Licenciatura en Educación Infantil Corporación Universitaria Minuto de Dios UNIMINUTO ( 2019)

La educación infantil, sugiere de manera inacabada pensar en las infancias como campo de estudios, contando con el niño sujeto social, contemporáneo, autodeterminado, autónomo y subjetivo, provisto de características en las que confluyen diversos aspectos que hacen parte del entorno con experiencias significativas, en la que se debe asumir la práctica pedagógica, teniendo en cuenta la diversidad de contextos, el desarrollo social en Colombia y modalidades educativas de carácter público y privado en contextos privados y rurales. Al igual, la familia se considera parte esencial en las prácticas formativas de los educadores infantiles y así se evidencia en sus narrativas, en la que confieren una relación íntima en el desarrollo del niño, sus relaciones vinculares y las situaciones preocupantes que indicaron en sus manifestaciones en función del entorno familiar y vulneración de sus derechos. 
La diversidad como tema de discusión ha permitido poner en relación la atención a la primera infancia desde un enfoque de derechos, sobre todo para las poblaciones excluidas que han sido temas sentidos en la escuela. La educación inclusiva, atribuye prácticas interesantes para el trabajo en las infancias, en su desarrollo integral que supone en el maestro una actitud conducente a la disminución de las barreras de acceso permanencia a la educación con calidad, el fomento de habilidades, el diseño pedagógico, de implementación y evaluación de procesos, donde se consideren las particularidades de cada niño o niña, reconociendo su esencia mediante las formas que se tienen de ser y estar en el mundo.

Para culminar, se defiende el postulado de pensarse en infancias, a través de las experiencias significativas, que enriquecen la labor formativa del maestro. Rescatar a través de voces las riquezas de lo vivido, permitir la realización de interpretaciones críticas de la experiencia, conlleva a interpretar realidades, interactuar con ellas, con la invitación de creer en mundos posibles a través de la práctica pedagógica como ese lugar de sentidos, de significados que solo pueden ser posibles con la acción, con la crítica propositiva y en anhelo de transformación de realidades.

\section{CONCLUSIONES}

En relación a los resultados y discusiones del presente estudio, se pudo ilustrar las siguientes conclusiones: 1) La Sistematización de experiencias como parte de un ejercicio investigativo de carácter social, que permitió conocer de manera importante las voces de los sujetos que participaron, para extraer elementos en los que se comprendieron los desarrollos de sus prácticas pedagógicas; 2) Se describió la experiencia general de la Corporación Universitaria Minuto de Dios UNIMINUTO, a través de las orientaciones normativas y modalidades de atención en la práctica pedagógica, que permitió relacionar elementos a considerar en los hallazgos desde las narrativas y hallazgos para comprender las infancias y el rol del educador infantil en este campo de estudios y 3) Se evidenciaron hallazgos alrededor de las unidades de sentido pedagógico con temas vinculantes como la influencia de las modalidades de atención educativa, las emociones que hacen parte del saber pedagógico y el sentido y significado del ser maestro. Fue importante, realizar aportaciones sobre las miradas actuales para pensarse en la infancia -infancias contando con las percepciones de los maestros en formación, teniendo en cuenta dos aspectos importantes que más rescatan, como la familia y diversidad y que desde una perspectiva fenomenológica propia de la experiencia asume un lugar preponderante en los contextos donde se desarrollan las prácticas pedagógicas.

\section{REFERENCIAS}

Ackesjö, H. S. y Persson S., The Educacional Positioning of the Preschool-Class at the Border between Social Education and Academic Demands: An Issue of Continuity in Swedish Early Education? http://dx.doi.org/10.15640/jehd.v5n1a19 Journal of Education and Human Development, 5(1): 182-196, (2016)

Ainscow, M., Haciendo que las escuelas sean más inclusivas: lecciones a partir del análisis de la investigación internacional. Revista de Educación Inclusiva 5(1), 39-49(2012)

Arendt, H., ¿Qué es la política? , Ed., 1-156, Paidos, Barcelona, España (1997).

Arrabal, A., L. Lázaro, L. M., El derecho a la Educación y Atención de la primer Infancia en América Latina, https://doi.org/10.5944/educxx1.16.1.719 (The Right To Early Childhood Education and Care in Latin América), Educación XX1, 16(1), 105-121, (2013)

Creswell, J. W., Qualitative inquiry and research design: choosing among five approaches, 3ㄹ Ed 1-448 SAGE Publications, Inc, California, United States of America (2013)

Corporación Universitaria Minuto de Dios UNIMINUTO (Colombia)., Informe de Autoevaluación con fines de Acreditación del programa de la Licenciatura en Educación Infantil; 1-338 (2019)

Corporación Universitaria Minuto de Dios UNIMINUTO (Colombia)., Proyecto Educativo Institucional del Sistema 1-103 (2014)

Coy, L., Rojas, C., ¡Un, dos, tres por los niños escondidos en el pasado! Hacia una bioarqueología de la infancia en Colombia, https://doi.org/10.22380/2539472X.1051, Revista Colombiana de Antropología 56(1): 215-243, (2020)

Higgins, C., Human conditions for teaching: The place of pedagogy in arendt's vita activa. Teachers College Record, 112(2), 407-445 (2010)

Fierro, C., Fortoul, B., Rosas, L., Transformando la práctica docente. Una propuesta basada en la investigación-acción 1-248. Maestro y enseñanza Paidos México (1999)

Gómez, M.A. y Alzate M.M., La infancia contemporánea, Revista Latinoamericana de Ciencias Sociales, 12 (1): 77-89 (2014)

Kaplan, S., Hertzog, N.B., Pedagogy for Early Childhood Gifted Education, https://doi.org/10.1177/1076217516644637, Gifted Child Today, 39(3), 134-139, (2016). 
Lyndon, H., Bertram, T. Brown, Z., y Pascal, C., Pedagogically mediated listening practices; the development of pedagogy through the development of trust, https://doi.org/10.1080/1350293X.2019.1600806, European Early Childhood Education Research Jornal, 27(3) 360-370 (2019)

MEN., 18583: Ministerio de Educación Nacional (Colombia) Por la cual se ajustan las características específicas de calidad de los programas de Licenciatura para la obtención, renovación o modificación del registro calificado, y se deroga la Resolución 2041 de 2016, 1-12, Bogotá- Colombia (2017)

Mejía, M., La sistematización empodera y produce saber y conocimiento 4ª Ed.,1-172, Dirección Fe y Alegría, Quito, Ecuador (2015)

Miner, R., Interpretive and critical phenomenological crime studies: A model design. The Qualitative Report, 17(27), 1-32. (2012).

Noguera, C.E., y Marín, D.L., Saberes, normas y sujetos: cuestiones sobre la práctica pedagógica, https://doi.org/10.1590/0104-4060.53866. Educar em Revista, (66), 37-56, (2017)

Oliveira, J. y Formosinho, J., Praxeological research in early childhood: a contribution to a social science of the social, https://doi.org/10.1080/1350293X.2012.737707, European Early Childhood Education Research Journal, 20(4),471-476, (2012)

Rodríguez, M. V., Reflexión sobre las Prácticas Educativas que Realizan los Maestros Universitarios: El Caso de la Facultad de Educación de UNIMINUTO, https://doi.org/10.4067/S0718-50062019000100109 Formación universitaria, 12(1), 109-120, (2019).

Salazar, M., y Botero, P., Política, Niñez y Contextos de vulnerabilidad, trazos y narrativas en un contexto local de Colombia, Pensar la infancia desde América Latina. Un estado de la cuestión, CLACSO Consejo Latinoamericano de Ciencias Sociales - Consejo Latino-americano de Ciencias Sociales, pp 133-157 Buenos Aires (2013)

Sousa, D.J., Pedagogical documentation: the search for children's voice and agency, https://doi.org/10.1080/1350293X.2019.1600807, European Early Childhood Education Research Journal, 27(3), 371384, (2019)

Ortega, P., La pedagogía crítica: Reflexiones entorno a sus prácticas y desafíos, https://doi.org/10.17227/01212494.31pys26.33, Pedagogía Y Saberes, (31), 26-33, Bogotá- Colombia (2009)

Paredes, J., Pensamiento epistémico y conocimiento social: emergencias y potencialidades en la investigación social, https://doi.org/10.7440/res48.2014.10, Revista de Estudios Sociales (48) 125-138 (2014)

Posada, J.J., y Briceño, P., Subjetividades Políticas de Paz en Experiencias del Secretariado Nacional de Pastoral Social, 14(1), $163-175$ (2016)

Taibo, L., Gutiérrez, C., Muzzio, E., Graves vulneraciones de derechos en la infancia y adolescencia: Variables de funcionamiento familiar, https://doi.org/10.11144/JAVERIANA.UPSY17-3.GVDI. Universitas Psychologica, 17(3), 1-13. (2018)

Torres, A., Producción de conocimiento desde la investigación crítica, Nómadas (40), 69-83, (2014)

Van M. M., Phenomenology and meaning attribution. Indo - Pacific Journal of Phenomenology, https://doi.org/10.1080/20797222.2017.1368253 (1), 1-12 (2017).

Vargas, C. G., El enfoque praxeológico, 1aㅡ Ed., 27-28, Corporación Universitaria Minuto de Dios UNIMINUTO, Bogotá, Colombia (2011)

Vargas, C.G., La cuestión del método en pedagogía praxeológica, 1-213. Corporación Universitaria Minuto de Dios. Bogotá, Colombia (2017)

Wacquant, L. y Akçaoğlu, A., Practice and symbolic power in Bourdieu: The view from Berkeley, https://doi.org/10.1177/1468795X16682145,Journal of Classical Sociology, 17(1), 55-69, (2017).

Winterbottom, C. y Mazzocco, P., Reconstructing teacher education: a praxeological approach to pre-service teacher education, https://doi.org/10.1080/1350293X.2014.975940, European Early Childhood Education Research Journal, 24(4),495-507, (2016) 